\title{
On a Generalised Fourier Transform of Instantons Over Flat Tori
}

\author{
H. Schenk \\ Physikalisches Institut, Universität Bonn, Nussallee 12, D-5300 Bonn 1, \\ Federal Republic of Germany
}

\begin{abstract}
Recently P. Braam pointed out that Nahm's adaption of the ADHM procedure to the case of monopoles equally well applies to instantons over flat tori, relating them to instantons over the first Brillouin zone. We show that this construction has an inverse. Hence the Nahm transform actually is a duality transform.
\end{abstract}

\section{Introduction}

It is an enticing thought that Bloch's analysis [1] might be the appropriate means for studying Yang-Mills fields over flat tori. Due to the non-linear character of the Yang-Mills equations, however, one may doubt that such a method is expedient. In this light it is a remarkable observation of Braam [2] that Nahm's considerations on the construction of monopoles [3] may be translated to instantons over flat tori. While Braam associates an instanton over the respective Brillouin zone $\hat{T}$ (dual torus) to every instanton over a torus $T$, we shall give a formulation of his observation which will allow us to prove the invertibility of this construction: after repeated application of the Nahm transform we recover the original instanton.

The ideas presented here may also be interpreted as an explicit version of some ideas of Mukai [4] if one takes into account the theorem of Donaldson, Yau, and Uhlenbeck [5] relating the stability of vector bundles to the existence of Hermitian-Einstein connections.

\section{Vector Bundles over Flat Tori}

We consider a four dimensional flat torus $T=\mathbb{R}^{4} / \Lambda$, defined by a lattice $\Lambda \subset \mathbb{R}^{4}$. Every vector bundle over $T$ may be described by matrix-multipliers. A matrixmultiplier $e$ of rank $n$ is defined to be a map $e: \mathbb{R}^{4} \times A \rightarrow G L(n, \mathbb{C}), e:(x, \lambda) \mapsto e(x, \lambda)$, satisfying the condition

$$
e(x, \lambda+\mu)=e(x+\mu, \lambda) e(x, \mu) .
$$


The associated vector bundle is obtained as a quotient of $\mathbb{R}^{4} \times \mathbb{C}^{n}$ over $\Lambda$, where the action of $\Lambda$ is given by $\lambda:(x, z) \mapsto(x+\lambda, e(x, \lambda) z)$ for every $\lambda \in \Lambda$. A section $S$ of this vector bundle may be identified with a function $S: \mathbb{R}^{4} \rightarrow \mathbb{C}^{n}$ obeying $S(x+\lambda)$ $=e(x, \lambda) S(x), \lambda \in \Lambda$, whereas connections behave as

$$
A(x+\lambda)=e(x, \lambda) A(x, \lambda) e^{-1}(x, \lambda)-e^{-1}(x, \lambda) d e(x, \lambda) .
$$

Remark. In what follows we restrict ourselves to unitary matrix-multipliers.

The Dirac operators we shall use in the sequel are $D: \Gamma\left(T, E \otimes S^{-}\right)$ $\rightarrow \Gamma\left(T, E \otimes S^{+}\right)$and $\bar{D}: \Gamma\left(T, E \otimes S^{+}\right) \rightarrow \Gamma\left(T, E \otimes S^{-}\right)$. Here $S^{ \pm}$are the trivial spinor bundles over $T$ corresponding to the trivial spin structure. Explicitly, the Dirac operators associated to a covariant derivative $\nabla$ are $D:=e_{\alpha}^{\dagger} \nabla_{\alpha}$ and $\bar{D}:=e_{\alpha} \nabla_{\alpha}$ with $e_{4}:=1$ and $e_{j}:=i \sigma_{j}, j=1,2,3$, the $\sigma_{j}$ being the Pauli matrices.

\section{Construction of the Dual Instanton}

On the torus $T=\mathbb{R}^{4} / \Lambda$ there exists a group of flat line bundles associated to the representations of the fundamental group $\pi_{1}(T) \cong \Lambda$, which are parametrised by the dual torus $\hat{T}:=\mathbb{R}^{4} / \Lambda^{*}$. These line bundles $\left\{L_{k}\right\}_{k \in \hat{T}}$ are given by constant matrix multipliers of the form $e(x, \lambda):=e^{i k \lambda}$.

A connection on a vector bundle $E$ over $T$ induces a connection on the twisted vector bundle $E \otimes L_{k}, k \in \hat{T}$. If the connection on $E$ is self-dual and irreducible in the sense that the structure group cannot be compressed to $U(r) \times U(n-r), 0<r<n$, then the same holds for the induced connection on $E \otimes L_{k}$. From the irreducibility it follows that $E \otimes L_{k}$ has no non-zero covariant constant sections. Next we observe that for an irreducible self-dual connection the Dirac operator $D^{k}$ associated to the induced connection on $E \otimes L_{k}$ has a trivial cokernel, $\operatorname{coker} D^{k}=0$. To prove this recall the identities coker $D^{k}=\operatorname{ker} \bar{D}^{k}, \operatorname{ker} \bar{D}^{k}=\operatorname{ker} D^{k} \bar{D}^{k}$, and upon using self-duality $D^{k} \bar{D}^{k}=\Delta^{k}$. Here $\Delta^{k}$ denotes the covariant laplacian operating on the sections of $E \otimes L_{k} \otimes S^{+}$. It is obvious that an element of coker $\bar{D}^{k}$ corresponds to a covariant constant section of $E \otimes L_{k}$. According to the above there is no such section besides the zero section. The Atiyah Singer index theorem [6] now yields that $\operatorname{dimker} D^{k}$ is in fact constant, i.e. it is independent of $k \in \hat{T}$ : because of index $D^{k}=\operatorname{dimker} D^{k}$ and $c_{2}\left(E \otimes L_{k}\right)[T]=c_{2}(E)[T]$ we obtain $-\operatorname{dimker} D^{k}=c_{2}(E)[T]$. Due to this

$$
E:=\bigcup_{k \in \hat{T}}\{k\} \times \operatorname{ker} D^{k}
$$

defines a vector bundle over the dual torus $\hat{T}$ called the bundle dual to $E$. In order to get more explicit formulae it is convenient and completely equivalent to the preceding description to work with a family of connections on the fixed vector bundle $E$. The family of connections is obtained by substituting $A \mapsto A^{k}:=A-i k_{\alpha} d x_{\alpha}, k \in \mathbb{R}^{4}$. We denote the Dirac operator associated to $A^{k}$ again by $D^{k}$. In terms of sections the change of description is just multiplying the sections of $E \otimes L_{k}$ with $e^{-i k x}$.

Because the pull-back of the dual bundle $\hat{E}$ by $\varrho: \mathbb{R}^{4} \mapsto \mathbb{R}^{4} / \Lambda^{*}$ is trivial, it is possible to find an orthonormalised family of sections $\left\{U_{j}(x, k)\right\}_{j \in J}$ of $E$ forming a basis of $k e r D^{k}$ and smoothly depending on $k \in \mathbb{R}^{4}$. Note, for fixed $k \in \mathbb{R}^{4}$ and $\mu \in \Lambda^{*}$ the basis $\left\{e^{-i \mu x} U_{j}(x, k+\mu)\right\}_{j \in J}$ is equivalent to the one given above. In matrix 
notation this equivalence reads

$$
\begin{aligned}
U(x, k):= & \left(U_{1}(x, k), \ldots, U_{v}(x, k)\right),-v:=c_{2}(E)[T], \\
& e^{-i \mu k} U(x, k+\mu)=U(x, k) a^{\dagger}(k, \mu) .
\end{aligned}
$$

The matrices $a(k, \mu)$ appearing in this formula actually form a unitary matrix multiplier defining the dual bundle $\hat{E}$. Stated differently, $e^{i k x} U(x, k)$ is the isomorphism that maps the fibre $\hat{E}_{k}$ of $\hat{E}$ over $k \in \hat{T}$ to $\operatorname{ker} D^{k} C \Gamma\left(T, E \otimes L_{k} \otimes S^{-}\right)$. Upon using this isomorphism we obtain a canonical connection $\hat{A}$ on $\hat{E}$. This connection is explicitly given by the formula

$$
\hat{A}_{\alpha}(k):=\int_{T} d x U^{\dagger}(x, k) \frac{\partial}{\partial k_{\alpha}} U(x, k) .
$$

The important fact about the canonical connection on $\hat{E}$ is the following observation of P. Braam.

Theorem (P. Braam). The canonical connection $\hat{A}$ on the bundle $\hat{E}$ dual to E is selfdual.

Proof. Upon using the Greens function of $\Delta^{k}=D^{k} \bar{D}^{k}$,

$$
\Delta^{k} G(x, y ; k)=-\delta(x, y),
$$

and the projection onto the orthogonal space of $\operatorname{ker} D^{k}$,

$$
\bar{D}^{k} G(x, y ; k) D^{k}=-\delta(x, y)+U(x, k) U^{\dagger}(y, k),
$$

it is straightforward to compute the curvature of $\hat{A}$,

$$
F_{\alpha \beta}(k)=2 i \int_{T \times T} d x d y U^{\dagger}(x, k) \bar{\eta}_{\alpha \beta} G(x, y ; k) U(y, k),
$$

with $e_{\alpha} e_{\beta}^{\dagger}=\delta_{\alpha \beta}+i \bar{\eta}_{\alpha \beta}$. The self-duality of $\hat{F}(k)$ now is a direct consequence of the selfduality of $\bar{\eta}_{\alpha \beta}$.

Note. The proof requires the commutativity of $D^{k} \bar{D}^{k}$ with all the $e_{\alpha}$, which is equivalent to the self-duality of $A$.

Collecting the results yields the following theorem.

Theorem 1. Let $(E, A)$ be an unitary vector bundle $E$ over a flat four dimensional torus $T$ endowed with an irreducible self-dual connection $A$ and with $c_{1}(E)=0$. Then the kernel of the associated family of Dirac operators $\left\{D^{k}\right\}_{k \in \hat{T}}$ defines a unitary vector bundle $\hat{E}$ over the dual torus $\hat{T}$ with $c_{1}(\hat{E})=0$, $\operatorname{rank} \hat{E}=-c_{2}(E)[T]$ and $-c_{2}(\hat{E})[\hat{T}]=\operatorname{rank} E$ if $\operatorname{rank} \hat{E} \geqq 2$. Furthermore there exists a canonical self-dual connection $\hat{A}$ on $\hat{E}$.

Remark. The relations between the Chern classes follow from the index theorem for families of operators [6].

Definition. The datum $(\hat{E}, \hat{A})$ is called the Nahm transform of $(E, A)$.

\section{The Inversion of the Nahm Transform}

This section is devoted to the proof of the following theorem. 
Theorem 2.1. The Nahm Transform is invertible in the sense that it can be applied to $(\hat{E}, \hat{A})$ leading back to the original datum $(E, A)$.

As a corollary we shall obtain the subsequent corollary.

Corollary 2.2. The Nahm transform maps irreducible self-dual connections to irreducible self-dual connections.

The first part of the proof of Theorem 2.1 is checking that the kernel of the dual Dirac operator yields a vector bundle over the original torus $T$. By dual Dirac operator we mean the Dirac operator $\hat{D}$ associated to the connection $\hat{A}$ on $\hat{E}$ over $\hat{T}$. Notice that the flat line bundles over $\hat{T}$ are parametrised by $T$, so that the family of operators we now have to dual with is $\left\{\hat{D}^{x}\right\}_{x \in T}$.

Lemma 2.3. Let $\chi(k, x)$ be defined by

$$
\pi \tilde{\chi}(k, x)=v(T)^{1 / 2} e^{i k x} \int_{T} d y U^{\dagger}(y, k) G(y, x ; k),
$$

with $v(T)$ being the volume of $T$ and $\tilde{\chi}$ the spinor dual to $\chi$,

$$
\tilde{\chi}=\chi^{t_{\varepsilon}}, \quad \varepsilon:=\left(\begin{array}{cc}
0 & 1 \\
-1 & 0
\end{array}\right) .
$$

Then $e^{-i k x} \chi(k, x)$ is an isomorphism that maps the fibre $E_{x} \subset E, x \in T$ to $\operatorname{ker} \hat{D}^{*}$ $c \Gamma\left(\hat{T}, \hat{E} \otimes \hat{L}_{x} \otimes \hat{S}^{-}\right)$.

Obviously $e^{-i k x} \chi(k, x)$ is well defined as an homomorphism from $E_{x}$ to $\Gamma\left(\widehat{T}, \hat{E} \otimes \hat{L}_{x} \otimes \hat{S}^{-}\right)$. Because of $e_{\alpha}^{\dagger}=-\varepsilon\left(e_{\alpha}^{t}\right) \varepsilon$, the claim that its image lies in $\operatorname{ker} \hat{D}^{x}$ is equivalent to

$$
\hat{\nabla}_{\alpha} \int_{T} d y U^{\dagger}(y, k) G(y, x ; k) e_{\alpha}=0,
$$

with $\hat{\nabla}$ denoting the covariant derivative with respect to $\hat{A}$. Upon using

$$
\int_{T} d x \hat{\nabla}_{\alpha} U^{\dagger}(x, k) \varphi(x)=-i \int_{T \times T} d y d x U^{\dagger}(y, k) G(y, x ; k) e_{\alpha} D^{k} \varphi(x),
$$

$\varphi \in \Gamma\left(T, E \otimes S^{-}\right)$and the definition of the Greens function this relation is easily verified. It remains to prove bijectivity.

Lemma 2.4. The homomorphism defined by $e^{-i k x} \chi(k, x)$ is injective.

Proof. It suffices to show

$$
\int d k \chi^{\dagger}(k, x) \chi(k, x)=i d_{E_{x}} .
$$

From the definition of $\chi(k, x)$ and Eq. (1.4) we deduce

$$
\chi^{\dagger}(k, x) \chi(k, y)=-(2 \pi)^{-2} v(T) e^{i k(y-x)} \partial_{k}^{2} G(x, y ; k),
$$

with

$$
\partial_{k}^{2}:=\frac{\partial}{\partial k_{\alpha}} \frac{\partial}{\partial k_{\alpha}} .
$$

Recalling the singular behaviour of the Greens function we then find

$$
\lim _{y \rightarrow x} \int_{\hat{T}} d k e^{i k(x-y)} \partial_{k}^{2} G(x, y ; k)=4 \pi^{2} v(T)^{-1} \mathrm{id}_{E_{x}} .
$$


Together with the familiar relation between the volumina of $T$ and $\hat{T}, v(T) v(\hat{T})$ $=(2 \pi)^{4}$ this completes the proof.

To demonstrate surjectivity is a little more laborious. Once we know that the laplacian $\hat{\Delta}^{x}$ operating on the sections of $\hat{E} \otimes \hat{L}_{x} \otimes S^{+}$is invertible we have the following simple lemma.

Lemma 2.5. The homomorphism defined by $e^{-i k x} \chi(k, x)$ is surjective if $\hat{\Delta}^{x}$ is invertible.

Proof. From the preceding lemma we know that the dimension of the image of the homomorphism is rank $E$. Due to the self-duality of $\hat{A}$ the Dirac operator $\hat{D}^{x}$ has coker $\hat{D}^{x}=0$ if $\operatorname{ker} \hat{\Delta}^{x}=0$. Thus, using the index theorem we find that the dimension of the target space $\operatorname{ker} \hat{D}^{x}$ also equals rank $E$.

The technical part of our reasoning is the construction of the Greens function of $\hat{A}^{x}$. From (2.6) we can read off how to do this.

Lemma 2.6. The Greens functions

$$
\hat{\Delta}^{x} \widehat{G}(k, p ; x)=-\delta(k, p)
$$

of the laplacian $\hat{\Delta}^{x}$ is given by the formula

$$
\hat{G}(k, p ; x)=\frac{e^{i x(k-p)}}{4 \pi^{2}} \sum_{m \in \Lambda^{*}} \int_{T} d y e^{i m(x-v)} \frac{U^{\dagger}(y, k) U(y, p)}{(m+k-p)^{2}} .
$$

Proof. With minor modifications the proof follows well-known lines. All we need are two technical results.

$$
\left.\hat{\nabla}_{\alpha}^{x} \widehat{G}(k, p ; x)\right|_{p=k+\varepsilon}=-\frac{e^{-i x \varepsilon}}{2 \pi^{2}} \frac{\varepsilon_{\alpha}}{|\varepsilon|^{4}} \int_{T} d y U^{\dagger}(y, k+\varepsilon) U(y, k)+O\left(|\varepsilon|^{-2}\right)
$$

and

$$
\widehat{\Delta}^{x} \widehat{G}(k, p ; x)=0 \quad \text { if } \quad k-p \neq 0 \bmod \Lambda^{*} .
$$

To verify the first formula we have to insert the definitions, use (2.4) and

$$
D^{k+n} U(x, p)=-i e_{\alpha}^{\dagger}(n+k-p)_{\alpha} U(x, p) .
$$

The second formula is proved analogously by first deriving

$$
\hat{\Delta} U^{\dagger}(x, k)=-4 \pi v(T)^{-1 / 2} e^{-i k x} \tilde{\chi}(k, x),
$$

which again is a consequence of (2.4).

Lemma 2.5 and Lemma 2.6 complete the proof of Lemma 2.3. Now we are in the position to prove Theorem 2. What we have to show is

$$
A_{\alpha}(x)=\int_{T} d k \chi^{\dagger}(k, x) \frac{\partial}{\partial x_{\alpha}} \chi(k, x) .
$$

This is easily done using a formula similar to (2.4),

$$
\int_{\hat{T}} d k U(x, k) \widehat{\nabla}_{\alpha} \varphi(k)=-i \int_{\hat{T} \times T} d k d y e_{\beta} \nabla_{\beta} G(x, y ; k) e_{\alpha}^{\dagger} U(y, k) \varphi(k),
$$


with $\varphi(k) \in \Gamma(\hat{T}, \hat{E})$. Inserting the definition (2.1), applying the formula to $e^{-i k x} \tilde{\chi}(k, x) e_{\alpha}$ upon performing the tensor product with respect to the spinor space, and recalling (2.3) we find

$$
0=e_{\beta} \int_{T} d k e_{\alpha}^{\dagger} M_{\beta}(k, x) e_{\alpha}
$$

with

$$
M_{\beta}:=\left(\begin{array}{cc}
\left(\nabla_{\beta} \chi_{2}^{\dagger}\right) \chi_{2} & -\left(\nabla_{\beta} \chi_{1}^{\dagger}\right) \chi_{2} \\
-\left(\nabla_{\beta} \chi_{2}^{\dagger}\right) \chi_{1} & \left(\nabla_{\beta} \chi_{1}^{\dagger}\right) \chi_{1}
\end{array}\right) .
$$

The identity $e_{\alpha}^{\dagger} M e_{\alpha}=2\left(\operatorname{tr}_{2} M\right) e_{4}$ (with $\operatorname{tr}_{2}$ denoting the trace with respect to the spinor space) now leads to the desired result,

$$
0=e_{\beta} \int_{\hat{T}} d k\left[\left(\nabla_{\beta} \chi_{1}^{\dagger}\right) \chi_{1}+\left(\nabla_{\beta} \chi_{2}^{\dagger}\right) \chi_{2}\right](k, x) .
$$

Next we show that the Nahm transform preserves irreducibility.

Proof of Corollary 2.2. Suppose $(E, A)$ is irreducible and mapped by the Nahm transform to a reducible $(\widehat{E}, \hat{A})$. At least after applying a gauge transformation we may assume that $\hat{E}$ decomposes into a direct sum with $\hat{A}$ restricting to a self-dual connection on each component. Because of Lemma 2.6 the kernel of the associated family of Dirac operators defines a vector bundle over $T$ endowed with a self-dual connection when restricted to one of the components of $\hat{E}$. According to Theorem 2.1 this means that $(E, A)$ is up to a gauge transformation split, contradicting the assumption of its irreducibility.

Finally it follows from Lemma 2.6 that there exists no 't Hooft-like instanton [7] on a flat torus.

Corollary 2.7. On a vector bundle E over a flat torus with $\operatorname{rank} E \geqq 2, c_{1}(E)=0$, and $c_{2}(E)[T]=-1$ there exists no irreducible self-dual connection.

Proof. Suppose there is such a connection $A$ on $E$. Then the Nahm transform of $(E, A)$ is a flat line bundle over $\hat{T}$ endowed with a flat connection. As a consequence $\widehat{\Delta}^{x}$ has a one dimensional kernel for a particular $x \in T$. This is incompatible with Lemma 2.6.

\section{Conclusions}

The Nahm transform is an invertible map from the space of instantons over a flat torus to the instantons over the dual torus. It preserves irreducibility and permutes rank and instanton number. Together with theorem of Taubes [8] this property proves the existence of $c_{2}(E)[T]=-2$ instantons for $S U(n)$ with $n \geqq 4$.

The formulae used to describe the Nahm transform explicitly are very similar to those of the ADHM construction [7] and in particular to the considerations on "reciprocity" of Goddard and Corrigan [9].

It is interesting to note that the local version of the index theorem obtained by the usual $\zeta$-function regularization method,

$$
\frac{\partial}{\partial k_{\alpha}} \hat{J}_{\alpha}^{5}(k)=\frac{1}{8 \pi^{2}} * \operatorname{tr}[\hat{F} \wedge \hat{F}](k)+\frac{2}{v(T)} \int_{T} d x \operatorname{tr} \chi^{\dagger}(k, x) \chi(k, x),
$$


with

$$
\hat{J}_{\alpha}^{5}(k)=\frac{1}{i v(T)} \int_{T} d x \operatorname{tr}\left(\nabla_{\alpha} \chi^{\dagger}(k, x)\right) \chi(k, x)
$$

leads after some formal manipulation to

$$
* \operatorname{tr}[\hat{F} \wedge \hat{F}](k)=-\frac{1}{v(T)} \int_{T} d x \lim _{y \rightarrow x} \operatorname{tr} \partial_{k}^{2} \partial_{k}^{2} \ln G(x, y ; k) .
$$

This relation seems to be remarkable because it is the regularised version of "*tr $[\hat{F} \wedge \hat{F}](k)=\partial_{k}^{2} \partial_{k}^{2} \ln \operatorname{Det}\left(\Delta^{k}\right)$." Comparison with recent results on elliptic families [10] suggests that this relation should admit a geometric interpretation, thus providing us with a deeper insight into the nature of Nahm's transform.

Acknowledgement. First of all I would like to thank P. Braam and P. Kronheimer for introducing me to their beautiful ideas. I thank Prof. W. Nahm for a number of interesting discussions and Prof. K. Meetz for his careful reading of the manuscript and his continuous support.

\section{References}

1. Ashcroft, N., Mermin, D.: Solid state physics. New York: Holt, Rinehard and Winston 1976

2. Braam, P.: Private communication

3. Nahm, W.: Monopoles in quantum theory. In: Proceedings of the Monopole Meeting, Trieste, Italy, Dec. 1981. Cragie, N.S., Goddard, P., Nahm, W. (eds.). Singapore: World Scientific 1982

4. Mukai, S.: Duality between $D(X)$ and $D(\hat{X})$ with its application to Picard sheaves. Nagoya Math. J. 81, 153-175 (1981)

5. Donaldson, S., Yau, S.-T., Uhlenbeck, K.: Anti-self-dual Yang-Mills connections over complex algebraic surfaces and stable vector bundles. Proc. Lond. Math. Soc. (3) 50, 1-26 (1981); Uhlenbeck, K., Yau, S.-T.: Hermitian-Einstein connections in stable vector bundles. Commun. Pure Appl. Math. Suppl. 39(S), 257-293 (1986)

6. Atiyah, M.F., Singer, I.: Proc. Natl. Acad. Sci. USA 81, 2597-2600 (1984)

7. Atiyah, M.F.: Geometry of Yang-Mills fields. Lenzioni fermiane, Accademia Nazionale Dei Lincei Suola Normale Superiore, Pisa 1979

8. Taubes, C.H.: Self-Dual connections on 4-manifolds with indefinite intersection matrix. J. Differ. Geom. 19, 517-560 (1984)

9. Goddard, P., Corrigan, E.: Construction of instanton and monopole solutions and reciprocity. Ann. Phys. 154, 253--279 (1984)

10. Bismuth, J.-M., Freed, D.S.: The analysis of elliptic families. I. Metrics and connections on determinant line bundles. Commun. Math. Phys. 106, 159-176 (1986)

Communicated by C. H. Taubes

Received October 6, 1986; in revised form September 21, 1987 
